\title{
Isotope anomalies produced by extremely small isotope fractionations: A process-driven amplification effect?
}

\author{
YINING ZHANG ${ }^{1,2}$, YUN LIU $^{1}$
}

${ }^{1}$ State Key Labrotary of Ore Deposit Geochemistry, Institute of Geochemisry, Chinese Academy of Sciences, Guiyang, China (E-mail: zhangyining17@mails.ucas.edu.cn, liuyun@vip.gyig.ac.cn)

${ }^{2}$ University of Chinese Academy of Sciences, Beijing, China

According to the classic isotope fractionation theory developed by Urey and Bigeleisen [1,2], isotope anomalies (e.g., $\Delta^{17} \mathrm{O}, \mu^{142} \mathrm{Nd}$ and $\mu^{182} \mathrm{~W}$ ) observed in mantle-derived samples are generally attributed to radioactive decay or nucleosynthetic rather than fractionations $[3,4]$. However, the discovery of the nuclear field shift effect (NFSE) shed new light on the orgin of such mass-independent isotope anomalies [5,6]. At the same time, because of the small magnitudes of such anomalies, it is quite necessary to check how such NFSE-caused fractionation changes during the formation of mantel-derived samples.

Inspired by previous works [7,8], here we devloped a multi-stage closed-system melting and crystallization evolution model (MC2-model) and conduct Monte Carlo simulations to trace the changes of NFSE-caused fractionations. A high-temperature approximation developed by [6] and exponential law were used to calculate the isotope fractionation factors and final measured isotope anomalies, respectively.

Our simulation work [9] show that there exists an amplification effect for such NFSE-caused fractionations during such multi-stage processes. Such effect scales linearly with the stage number $N$, the total times of melting and crystallization. Combined with statistical analysis, our results indicate that some ppm-level isotope anomalies observed may just extremely small NFSE-caused fractionations magnified after multi-stage high-temperature processes.

[1] Urey, H. C., J. Chem. Soc., 1947, 562-581. [2] Bigeleisen, J., J. Chem. Phys., 1947, 15, 261-267. [3] Rizo, H., et al., Geochem. Cosmochim. Acta, 2016, 175, 319-336. [4] Boyet, M., et al., Earth Planet. Sci. Lett., 2018, 488, 68-78. [5] Bigeleisen, J., J. Am. Chem. Soc., 1996, 118, 3676-3680. [6] Fujii, T., et al., Earth Planet. Sci. Lett., 2006, 247, 1-9. [7] Liang, Y and Liu, B., Geochem. Cosmochim. Acta, 2016, 173, 181-197. [8] Liu, B and Liang Y., Geochem. Cosmochim. Acta, 2017, 203, 216-234. [9] Zhang, Y and Liu Y., under Review. 